hep-th/0409018

IFT-P.041/2004

MIT-CTP-3537

\title{
WZW-like Action for Heterotic String Field Theory
}

\author{
Nathan Berkovits $^{1}$, Yuji Okawa ${ }^{2}$, and Barton Zwiebach ${ }^{2}$ \\ 1 Instituto de Física Teórica \\ Universidade Estadual Paulista \\ R. Pamplona 145, São Paulo, SP 01405-900, BRASIL \\ nberkovi@ift.unesp.br \\ ${ }^{2}$ Center for Theoretical Physics \\ Massachusetts Institute of Technology \\ Cambridge, MA 02139, USA \\ okawa@lns.mit.edu, zwiebach@lns.mit.edu
}

\begin{abstract}
We complete the construction of the Neveu-Schwarz sector of heterotic string field theory begun in hep-th/0406212 by giving a closed-form expression for the action and gauge transformations. Just as the Wess-Zumino-Witten (WZW) action for open superstring field theory can be constructed from pure-gauge fields in bosonic open string field theory, our heterotic string field theory action is constructed from pure-gauge fields in bosonic closed string field theory. The construction involves a simple alternative form of the WZW action which is consistent with the algebraic structures of closed string field theory.
\end{abstract}




\section{Contents}

1 Introduction $\quad 2$

2 Open superstring field theorv 4

2.1 Rewriting the WZW action . . . . . . . . . . . . . . . . . 4

2.2 Open superstring field theorv $\ldots \ldots \ldots \ldots \ldots \ldots \ldots$

\begin{tabular}{|lr}
3 Heterotic string field theorv & 8
\end{tabular}

3.1 Shifted structures in closed string field theorv . . . . . . . . . . . . . . . . . . 9

3.2 Pure-gauge closed string fields . . . . . . . . . . . . . . . . . . . . . . . . 11

3.3 Derivations and associated string fields . . . . . . . . . . . . . . . . . . . . 12

3.4 Heterotic string field theorv action . . . . . . . . . . . . . . . . . . . . . 14

3.5 Reparameterization invariance and linear homotopv $\ldots \ldots \ldots$. . . . . . . . . . 16

4 Heterotic string action in standard WZW form $\quad 17$

5 Conclusions $r$

\section{Introduction}

In bosonic open string field theory, the first-quantized condition $Q A=0$ for physical vertex operators is generalized to the nonlinear equation of motion

$$
Q A+A A=0
$$

where string fields are multiplied using the open string star product [1]. To extend this result to the Neveu-Schwarz (NS) sector of open superstring field theory, an equation of motion $Q A+Z(A A)=0$ was proposed where $Z$ is a picture-raising operator inserted at the string midpoint [2]. Approaches involving midpoint picture-changing insertions, however, have contact-term problems which lead to a breakdown of gauge invariance 3 .

This difficulty is resolved in open superstring field theory by working in the 'large' Hilbert space of Friedan, Martinec, and Shenker [4], which includes the $\xi$ zero mode coming from bosonization of the $(\beta, \gamma)$ ghosts. Vertex operators $\Phi$ in the large Hilbert space are related to vertex operators $A$ in the small Hilbert space by $\eta \Phi=A$ where $\eta$ denotes the zero mode of the field conjugate to $\xi$. Therefore, the first-quantized condition $Q A=0$ implies that $\eta Q \Phi=0$ is the linearized equation of motion in the large Hilbert space. The operators $\eta$ and $Q$ anticommute and both of them square to zero.

The linearized equation for $\Phi$ generalizes to the nonlinear equation of motion

$$
\eta\left(e^{-\Phi} Q e^{\Phi}\right)=0
$$

which can be derived from a Wess-Zumino-Witten (WZW) action for open superstring field theory which does not involve explicit picture-changing operator insertions [5]. Note that the open superstring 
equation of motion of (1.2) can be written as $\eta \bar{A}_{Q}=0$ where $\bar{A}_{Q}=e^{-\Phi} Q e^{\Phi}$ is in fact a pure-gauge solution of the bosonic open string field theory equation of motion of (1.1).

In bosonic closed string field theory, the first-quantized condition for physical vertex operators $\Psi$ also takes the form $Q \Psi=0$, where $Q=Q_{L}+Q_{R}$, is the BRST operator obtained by adding the contributions from the left-moving $(L)$ and right-moving $(R)$ sectors. This equation is generalized to the following nonlinear equation of motion

$$
Q \Psi+\sum_{n=2}^{\infty} \frac{\kappa^{n-1}}{n !}\left[\Psi^{n}\right]=0,
$$

where string fields are multiplied using the various closed string products [6, 7] and $\kappa$ is the gravitational constant. Although one can extend this equation to heterotic strings by non-canonical insertions of picture-changing operators [8], it was recently shown by two of the authors that insertions of picturechanging operators can be altogether avoided in heterotic string field theory if one works in the large Hilbert space [9]. Denoting by $\eta=\eta_{L}$ the zero mode of the left-moving superghost, the first-quantized condition for NS heterotic vertex operators $V$ in the large Hilbert space takes the form $\eta Q V=0$. The authors constructed the first few terms in the nonlinear generalization of the heterotic equation of motion, action, and gauge transformations.

In this paper, we complete this construction by giving closed-form expressions for the complete equation of motion, action, and gauge transformations in the NS sector of heterotic string field theory. Just as $\eta \bar{A}_{Q}=0$ is the open superstring equation of motion where $\bar{A}_{Q}$ is a pure-gauge solution to bosonic open string field theory, we show that $\eta \bar{\Psi}_{Q}=0$ is the heterotic equation of motion where $\bar{\Psi}_{Q}$ is a pure-gauge solution to the bosonic closed string equation of motion of (1.3). The heterotic string field theory action takes a rather simple but apparently unfamiliar form of the WZW action.

The familiar form of the WZW action includes two terms: one is written in two-dimensional covariant language and the other, the WZ term, is elegantly written as a three-dimensional covariant expression [10]. In the form we have found, the action is given by just one term. This term involves integration over three dimensions, just like the WZ term but, as written, is not manifestly twodimensional nor three-dimensional covariant. Both for ordinary bosonic WZW models and for open superstring theory, this alternative form of the action is completely equivalent to the more familiar one. For the heterotic string, the non-standard form of the WZW action appears to provide the fundamental definition of the theory. The transformation to the familiar WZW action is not generally valid, although it is possible in the case where the string field is chosen to have 'linear homotopy' in the third dimension.

As we explain in detail, our construction uses the BRST operator $Q$ and three more operators: the zero mode $\eta$ of the superghost, the derivative $\partial_{t}$ along the third direction of WZW theory, and the variation $\delta$. In open superstring field theory these four operators are all derivations of the star algebra of open strings. In particular, $Q$ and $\eta$ appear in the action symmetrically. On the other hand, the four operators are not on the same footing in heterotic theory: $Q$ is not a derivation of all closed string products, while the other three are. In particular, $Q$ and $\eta$ play different roles and do not appear symmetrically in the action. 
In section 2 of this paper we rewrite the WZW action for open superstring field theory in the nonstandard form which generalizes to heterotic string field theory. In section 3 we use this non-standard form of the WZW action to construct an explicit action for heterotic string field theory and show that it implies the desired equation of motion and gauge invariances. We also show that the first few terms in the expansion of the action coincides with the terms constructed in [9]. Some of the structures in open string field theory are absent in closed string field theory, and the non-standard form of the WZW action does not require these additional structures. In section 4 we discuss the relationship of our heterotic action with the more familiar form for the WZW action. Finally, in section 5 we summarize our results and discuss some possible applications.

\section{Open superstring field theory}

In this section we show that the familiar action for WZW theory can be recast in a rather useful and simple way. We then exhibit a similar recasting for the WZW open superstring field theory action. Our construction of the heterotic string field theory action will be based on an analogous simple form. The analysis of open superstrings in this section will use the tools that generalize to heterotic strings.

\subsection{Rewriting the WZW action}

Consider bosonic WZW theory on a two-dimensional compact space with coordinates $z$ and $\bar{z}$. As usual, to write a natural action we introduce a three-dimensional space. The extra dimension can be parametrized by a coordinate $t \in[0,1]$ and we introduce a Lie-algebra valued field $\Phi(t, z, \bar{z})$. The field $\Phi$ is required to vanish at $t=0$ for all $z$ and $\bar{z}$. The original two-dimensional space is obtained for $t=1$. The WZW action is written in terms of a pure-gauge connection

$$
A_{i}=e^{-\Phi}\left(\partial_{i} e^{\Phi}\right), \quad i=t, z, \bar{z} .
$$

By construction, this connection is flat

$$
F_{i j}=\partial_{i} A_{j}-\partial_{j} A_{i}+\left[A_{i}, A_{j}\right]=0,
$$

and both $A_{z}$ and $A_{\bar{z}}$ vanish for $t=0$. The WZW action is now given as

$$
S=\frac{1}{2 g^{2}} \int d^{2} z \int_{0}^{1} d t \operatorname{Tr}\left(\partial_{t}\left(A_{z} A_{\bar{z}}\right)+A_{t}\left[A_{z}, A_{\bar{z}}\right]\right) .
$$

The integral over $t$ can be explicitly done for the first term inside the parentheses; it gives the familiar kinetic term defined on the original two-dimensional space. We now show that the above action can be rewritten as

$$
S=\frac{1}{g^{2}} \int d^{2} z \int_{0}^{1} d t \operatorname{Tr}\left(\left(\partial_{z} A_{t}\right) A_{\bar{z}}\right) .
$$

To prove this we begin with (2.3), expand the $t$ derivatives and use the vanishing of $F_{t z}$ and $F_{t \bar{z}}$ to trade the $t$ derivatives for $z$ and $\bar{z}$ derivatives:

$$
S=\frac{1}{2 g^{2}} \int d^{2} z \int_{0}^{1} d t \operatorname{Tr}\left(\left(\partial_{z} A_{t}\right) A_{\bar{z}}+A_{z}\left(\partial_{\bar{z}} A_{t}\right)+A_{t}\left[A_{z}, A_{\bar{z}}\right]\right) .
$$


Integrating by parts the $\bar{z}$ derivative and using $F_{z \bar{z}}=0$ we find

$$
\left.S=\frac{1}{2 g^{2}} \int d^{2} z \int_{0}^{1} d t \operatorname{Tr}\left(\left(\partial_{z} A_{t}\right) A_{\bar{z}}-\left(\partial_{z} A_{\bar{z}}\right) A_{t}\right)\right)=\frac{1}{g^{2}} \int d^{2} z \int_{0}^{1} d t \operatorname{Tr}\left(\left(\partial_{z} A_{t}\right) A_{\bar{z}}\right),
$$

as we wanted to show.

\subsection{Open superstring field theory}

The WZW open superstring field theory action is given by

$$
S=-\frac{1}{2 g^{2}} \int_{0}^{1} d t\left\langle\left\langle\partial_{t}\left(A_{\eta} A_{Q}\right)+A_{t}\left\{A_{Q}, A_{\eta}\right\}\right\rangle\right\rangle
$$

where $\{A, B\} \equiv A B+B A$. We also use $[A, B] \equiv A B-B A$, and more generally,

$$
[A, B\} \equiv A B-(-1)^{A B} B A,
$$

where string fields or operators in the exponent represent their Grassmann property, $0(\bmod 2)$ if Grassmann even and $1(\bmod 2)$ if Grassmann odd. The double brackets in (2.7) denote correlators defined in the large Hilbert space using the geometry of the star product and its iterated versions, and

$$
A_{Q}=e^{-\Phi(t)}\left(Q e^{\Phi(t)}\right), \quad A_{\eta}=e^{-\Phi(t)}\left(\eta e^{\Phi(t)}\right), \quad A_{t}=e^{-\Phi(t)}\left(\partial_{t} e^{\Phi(t)}\right),
$$

where $\Phi(0)=0$ and $\Phi(1) \equiv \Phi$. Here $\eta$ denotes the zero mode of the superghost field $\eta(z)$. If we take $\Phi(t)=t \Phi$, these string fields become

$$
A_{Q}=e^{-t \Phi}\left(Q e^{t \Phi}\right), \quad A_{\eta}=e^{-t \Phi}\left(\eta e^{t \Phi}\right), \quad A_{t}=e^{-t \Phi}\left(\partial_{t} e^{t \Phi}\right)=\Phi .
$$

The open superstring field theory action has been usually written using this specific form of $\Phi(t)$.

The definitions in (2.9) are all of the form

$$
A_{X}=e^{-\Phi(t)}\left(X e^{\Phi(t)}\right),
$$

where $X$ is a derivation of the star product of open string fields, namely, $X$ satisfies $X(A B)=$ $(X A) B+(-1)^{X A} A(X B)$ for arbitrary string fields $A$ and $B .{ }^{1}$ Indeed, $Q, \eta$, and $\partial_{t}$ are derivations. They are also derivations of the graded commutator (2.8): $X[A, B\}=[X A, B\}+(-1)^{X A}[A, X B\}$.

The string field $A_{Q}$ has an important interpretation. It is a pure-gauge open string field associated with the 'large' gauge parameter $\Phi(t)$ in bosonic open string field theory. In this theory the equation of motion takes the form $Q A+A A=0$ and the infinitesimal gauge transformations take the form $\delta_{\epsilon} A=Q \epsilon+[A, \epsilon]$. One can readily check that $A_{Q}$ satisfies the string field equation of motion: $Q A_{Q}+A_{Q} A_{Q}=0$.

If we expand the bosonic open string field theory action about $A_{Q}$, the new BRST operator $Q^{\prime}$ in the action acts as follows:

$$
Q^{\prime} B=Q B+\left[A_{Q}, B\right\}
$$

\footnotetext{
${ }^{1}$ We use $t$ for $\partial_{t}$ in indices. For example, $A_{t}=A_{\partial_{t}}$.
} 
The operator $Q^{\prime}$ squares to zero, is a derivation of the star product, and is also a derivation of the graded commutator. One can view the $Q^{\prime}$ action as that of a covariant derivative with gauge connection $A_{Q}$, accordingly, we write $\nabla_{Q} B \equiv Q^{\prime} B$. The language of field strengths is quite natural indeed. For any two derivations $X$ and $Y$ that commute or anticommute, one finds that the field strength $F_{X Y}$ vanishes:

$$
F_{X Y} \equiv X A_{Y}-(-1)^{X Y} Y A_{X}+\left[A_{X}, A_{Y}\right\}=0 .
$$

We use this notation for field strengths even when $X$ or $Y$ is the derivation $\delta$. For any derivation $X$ which commutes or anticommutes with $Q$ the following useful identity holds:

$$
X\left(Q^{\prime} B\right)-(-1)^{X} Q^{\prime}(X B)=\left[X A_{Q}, B\right\} .
$$

As in the case of the ordinary WZW action, the superstring field theory action (2.7) can also be written as

$$
S=-\frac{1}{g^{2}} \int_{0}^{1} d t\left\langle\left\langle\left(\eta A_{t}\right) A_{Q}\right\rangle\right\rangle .
$$

We will prove the equivalence of the two actions later. Note that $A_{\eta}$ no longer appears in (2.15).

The gauge invariance of (2.15) can be shown by computing the general variation $\delta S$. We can compute the variation of the integrand using the explicit forms of $A_{Q}$ and $A_{t}$ to find

$$
\delta\left\langle\left\langle\left(\eta A_{t}\right) A_{Q}\right\rangle\right\rangle=\partial_{t}\left\langle\left\langle\left(\eta A_{\delta}\right) A_{Q}\right\rangle\right\rangle .
$$

Note that the variation of the string field is encoded in $A_{\delta}$. This direct approach, however, is not available in heterotic string field theory, where the closed string fields corresponding to $A_{Q}$ and $A_{t}$ do not have simple expressions. Moreover, while all field strengths vanish for open superstrings, the heterotic string analogs do not all vanish. It is therefore useful to calculate $\delta S$ without relying on the explicit expressions of $A_{Q}$ and $A_{t}$ and to extract the minimum structures which ensure gauge invariance of the action. We find that gauge invariance of the action follows from the following three equations:

$$
\begin{aligned}
\partial_{t} A_{Q} & =Q^{\prime} A_{t}, \\
\delta A_{Q} & =Q^{\prime} A_{\delta}, \\
\eta A_{Q} & =-Q^{\prime} A_{\eta} .
\end{aligned}
$$

These equations take the form $X A_{Q}=(-1)^{X} \nabla_{Q} A_{X}$ (which follows from $F_{X Q}=0$ ), for $X=\partial_{t}, \delta$, and $\eta$. In the computation of $\delta S$ we also use the identity:

$$
Q^{\prime}\left(\delta A_{t}-\partial_{t} A_{\delta}+\left[A_{\delta}, A_{t}\right]\right)=0 .
$$

This identity, which states that $Q^{\prime}$ annihilates $F_{\delta t}$, is not independent. It follows from (2.17), (2.18), $\left[\delta, \partial_{t}\right]=0$, and (2.14):

$$
\begin{aligned}
0 & =\delta\left(\partial_{t} A_{Q}\right)-\partial_{t}\left(\delta A_{Q}\right) \\
& =\delta\left(Q^{\prime} A_{t}\right)-\partial_{t}\left(Q^{\prime} A_{\delta}\right) \\
& =Q^{\prime}\left(\delta A_{t}\right)+\left[\delta A_{Q}, A_{t}\right]-Q^{\prime}\left(\partial_{t} A_{\delta}\right)-\left[\partial_{t} A_{Q}, A_{\delta}\right] \\
& =Q^{\prime}\left(\delta A_{t}-\partial_{t} A_{\delta}+\left[A_{\delta}, A_{t}\right]\right) .
\end{aligned}
$$


We are now ready to compute the variation of the action (2.15). The variation of the integrand is

$$
\delta\left\langle\left\langle\left(\eta A_{t}\right) A_{Q}\right\rangle\right\rangle=\left\langle\left\langle\left(\eta \delta A_{t}\right) A_{Q}\right\rangle\right\rangle+\left\langle\left\langle\left(\eta A_{t}\right) \delta A_{Q}\right\rangle\right\rangle .
$$

The first term on the right-hand side is evaluated as follows:

$$
\begin{aligned}
\left\langle\left\langle\left(\eta \delta A_{t}\right) A_{Q}\right\rangle\right\rangle & =-\left\langle\left\langle\delta A_{t}\left(\eta A_{Q}\right)\right\rangle\right\rangle=\left\langle\left\langle\delta A_{t}\left(Q^{\prime} A_{\eta}\right)\right\rangle\right\rangle=-\left\langle\left\langle\left(Q^{\prime} \delta A_{t}\right) A_{\eta}\right\rangle\right\rangle \\
& =-\left\langle\left\langle\left(Q^{\prime}\left(\partial_{t} A_{\delta}-\left[A_{\delta}, A_{t}\right]\right)\right) A_{\eta}\right\rangle\right\rangle=\left\langle\left\langle\left(\partial_{t} A_{\delta}-\left[A_{\delta}, A_{t}\right]\right)\left(Q^{\prime} A_{\eta}\right)\right\rangle\right\rangle \\
& =-\left\langle\left\langle\left(\partial_{t} A_{\delta}-\left[A_{\delta}, A_{t}\right]\right)\left(\eta A_{Q}\right)\right\rangle\right\rangle \\
& =\left\langle\left\langle\left(\eta \partial_{t} A_{\delta}\right) A_{Q}\right\rangle\right\rangle+\left\langle\left\langle\left[A_{\delta}, A_{t}\right]\left(\eta A_{Q}\right)\right\rangle\right\rangle .
\end{aligned}
$$

The second term on the right-hand side is evaluated as follows:

$$
\begin{aligned}
\left\langle\left\langle\left(\eta A_{t}\right) \delta A_{Q}\right\rangle\right\rangle & =\left\langle\left\langle\left(\eta A_{t}\right)\left(Q^{\prime} A_{\delta}\right)\right\rangle\right\rangle=\left\langle\left\langle\left(Q^{\prime}\left(\eta A_{t}\right)\right) A_{\delta}\right\rangle\right\rangle \\
& =-\left\langle\left\langle\left(\eta\left(Q^{\prime} A_{t}\right)\right) A_{\delta}\right\rangle\right\rangle+\left\langle\left\langle\left[\eta A_{Q}, A_{t}\right] A_{\delta}\right\rangle\right\rangle \\
& =-\left\langle\left\langle\left(\eta \partial_{t} A_{Q}\right) A_{\delta}\right\rangle\right\rangle+\left\langle\left\langle\left[\eta A_{Q}, A_{t}\right] A_{\delta}\right\rangle\right\rangle \\
& =-\left\langle\left\langle\partial_{t} A_{Q}\left(\eta A_{\delta}\right)\right\rangle\right\rangle+\left\langle\left\langle\left[\eta A_{Q}, A_{t}\right] A_{\delta}\right\rangle\right\rangle \\
& =\left\langle\left\langle\left(\eta A_{\delta}\right) \partial_{t} A_{Q}\right\rangle\right\rangle-\left\langle\left\langle\left[A_{\delta}, A_{t}\right]\left(\eta A_{Q}\right)\right\rangle\right\rangle .
\end{aligned}
$$

Therefore, the variation of the integrand of the action is given by (2.16). Since the variation of the integrand of the action is a total derivative in $t$, the variation of the action is given by

$$
\delta S=-\frac{1}{g^{2}}\left\langle\left\langle\left(\eta \bar{A}_{\delta}\right) \bar{A}_{Q}\right\rangle\right\rangle
$$

where the bar denotes evaluation at $t=1$, namely, $\bar{F} \equiv F(t=1)$. Using (2.19), the variation of the action can also be written as

$$
\delta S=\frac{1}{g^{2}}\left\langle\left\langle\left(\overline{Q^{\prime}} \bar{A}_{\delta}\right) \bar{A}_{\eta}\right\rangle\right\rangle .
$$

Note that $A_{\delta}$ depends on $t$ only through $\Phi(t)$ and $\delta \Phi(t)$. Therefore the relation between $A_{\delta}$ and $\delta \Phi(t)$ is invertible. Since ${\overline{Q^{\prime}}}^{2}$ and $\eta^{2}$ vanish, the action is invariant under variations that satisfy

$$
\bar{A}_{\delta}=\overline{Q^{\prime}} \Lambda^{\prime}+\eta \Omega .
$$

Therefore, we define gauge transformations for $\Phi(t)$ by

$$
A_{\delta}=Q^{\prime} \Lambda^{\prime}(t)+\eta \Omega(t)
$$

where $\Lambda^{\prime}(0)=0, \Lambda^{\prime}(1)=\Lambda, \Omega(0)=0$, and $\Omega(1)=\Omega$. Using the explicit forms of $A_{\delta}$ and $A_{Q}$, this equation can be written as

$$
\begin{aligned}
e^{-\Phi(t)}\left(\delta e^{\Phi(t)}\right) & =Q \Lambda^{\prime}(t)+e^{-\Phi(t)}\left(Q e^{\Phi(t)}\right) \Lambda^{\prime}(t)+\Lambda^{\prime}(t) e^{-\Phi(t)}\left(Q e^{\Phi(t)}\right)+\eta \Omega(t) \\
& =e^{-\Phi(t)}\left(Q\left(e^{\Phi(t)} \Lambda^{\prime}(t) e^{-\Phi(t)}\right)\right) e^{\Phi(t)}+\eta \Omega(t)
\end{aligned}
$$


Therefore, we have

$$
\delta e^{\Phi(t)}=(Q \Lambda(t)) e^{\Phi(t)}+e^{\Phi(t)}(\eta \Omega(t)),
$$

where $\Lambda(t)=e^{\Phi(t)} \Lambda^{\prime}(t) e^{-\Phi(t)}$ is a redefined gauge parameter. This is the usual form of the gauge transformations.

Let us next show the equivalence of this form of the action and the WZW action (2.7). In the case of the ordinary WZW action, we used three equations: $F_{t z}=0, F_{t \bar{z}}=0$, and $F_{z \bar{z}}=0$. Correspondingly, we also use three equations in the case of open superstring field theory. In addition to (2.17) and (2.19), which correspond to $F_{t Q}=0$ and $F_{\eta Q}=0$, respectively, we need the vanishing of $F_{t \eta}$ :

$$
\partial_{t} A_{\eta}-\eta A_{t}+\left[A_{t}, A_{\eta}\right]=0 .
$$

Using these three equations, the integrand of the action (2.7) can be rewritten in the following way:

$$
\begin{aligned}
& \frac{1}{2}\left\langle\left\langle\partial_{t}\left(A_{\eta} A_{Q}\right)\right\rangle\right\rangle+\frac{1}{2}\left\langle\left\langle A_{t}\left\{A_{\eta}, A_{Q}\right\}\right\rangle\right\rangle \\
= & \frac{1}{2}\left\langle\left\langle\left(\partial_{t} A_{\eta}+\left[A_{t}, A_{\eta}\right]\right) A_{Q}\right\rangle\right\rangle+\frac{1}{2}\left\langle\left\langle A_{\eta}\left(\partial_{t} A_{Q}\right)\right\rangle\right\rangle \\
= & \frac{1}{2}\left\langle\left\langle\left(\eta A_{t}\right) A_{Q}\right\rangle\right\rangle+\frac{1}{2}\left\langle\left\langle A_{\eta}\left(Q^{\prime} A_{t}\right)\right\rangle\right\rangle \\
= & \frac{1}{2}\left\langle\left\langle\left(\eta A_{t}\right) A_{Q}\right\rangle\right\rangle-\frac{1}{2}\left\langle\left\langle\left(\eta A_{Q}\right) A_{t}\right\rangle\right\rangle=\left\langle\left\langle\left(\eta A_{t}\right) A_{Q}\right\rangle\right\rangle .
\end{aligned}
$$

This completes the proof of equivalence.

We can view the construction of the action (2.15) in the following way. The action can be constructed from $A_{Q}$, which is a pure-gauge solution of bosonic open string field theory, and $A_{t}$, which satisfies the relation (2.17). The action is gauge invariant because $\delta A_{Q}$ and $\eta A_{Q}$ are $Q^{\prime}$ exact, and the gauge transformations (2.28) are consistent because $A_{\delta}$ depends on $t$ only through $\Phi(t)$ and $\delta \Phi(t)$.

Such string fields $A_{Q}, A_{t}$, and $A_{\delta}$ can be constructed by the following procedure. First, take the pure-gauge string field $e^{-\Phi}\left(Q e^{\Phi}\right)$ and define $A_{Q} \equiv e^{-\Phi(t)}\left(Q e^{\Phi(t)}\right)$, where we have replaced $\Phi$ by $\Phi(t)$. Since $A_{Q}$ is pure-gauge for arbitrary $\Phi(t)$, its variation can be written as an infinitesimal gauge transformation: $\delta A_{Q}=Q^{\prime} A_{\delta}$, where $A_{\delta}$ is the gauge parameter. Furthermore, $A_{\delta}$ is guaranteed to depend on $t$ only through $\Phi(t)$ and $\delta \Phi(t)$ because $A_{Q}$ and $Q^{\prime}$ depend on $t$ only through $\Phi(t)$. In open superstring field theory, such an $A_{\delta}$ can be found explicitly and is given by $e^{-\Phi(t)}\left(\delta e^{\Phi(t)}\right)$. The verification that this expression for $A_{\delta}$ satisfies $\delta A_{Q}=Q^{\prime} A_{\delta}$ relies only on the derivation property of $\delta$ and the commutativity of $\delta$ and $Q$. Since $\partial_{t}$ and $\eta$ are also derivations that commute or anticommute with $Q$, one can construct $A_{t}$ and $A_{\eta}$ satisfying (2.17) and (2.19) by simply replacing $\delta$ in $A_{\delta}$ with $\partial_{t}$ and $\eta$, respectively. We will apply this strategy to heterotic string field theory in the next section.

\section{$3 \quad$ Heterotic string field theory}

In this section we first develop the closed string field theory structures that are needed to construct the field theory of heterotic strings [1]. As opposed to the open string theory case, we have no concise expression for a pure-gauge closed string field. We build the pure-gauge closed string field with a 
finite gauge parameter $V$ by integrating infinitesimal gauge transformations. The procedure we follow gives a pure-gauge closed string field $G(V)$ associated with $V$ that is analogous to the open string pure-gauge field $e^{-\Phi}\left(Q e^{\Phi}\right)$ associated with $\Phi$. We then define the closed string field $\Psi_{Q} \equiv G(V(t))$, just as we defined $A_{Q} \equiv e^{-\Phi(t)}\left(Q e^{\Phi(t)}\right)$.

Our next step is the construction of closed string fields $\Psi_{t}, \Psi_{\delta}$, and $\Psi_{\eta}$ which satisfy

$$
\begin{aligned}
\partial_{t} \Psi_{Q} & =Q^{\prime} \Psi_{t}, \\
\delta \Psi_{Q} & =Q^{\prime} \Psi_{\delta}, \\
\eta \Psi_{Q} & =-Q^{\prime} \Psi_{\eta},
\end{aligned}
$$

where $Q^{\prime}$ is the BRST operator of the bosonic closed string field theory action expanded about $\Psi_{Q}$. The closed string fields $\Psi_{t}, \Psi_{\delta}$, and $\Psi_{\eta}$ are analogous to the open string fields $A_{t}$, $A_{\delta}$, and $A_{\eta}$, respectively. Finally, we propose the following string field theory action

$$
S=\frac{2}{\alpha^{\prime}} \int_{0}^{1} d t\left\langle\eta \Psi_{t}, \Psi_{Q}\right\rangle
$$

and we show that it has two gauge invariances, one generated by $Q$ and one generated by $\eta$. Moreover, when expanded in powers of the gravitational constant $\kappa$, the action (3.4) reproduces the kinetic term and all the interaction terms determined in 9. The action (3.4) is a central result in this paper. It can be said to be a WZW-like action since it takes a form analogous to the WZW action (2.4).

\subsection{Shifted structures in closed string field theory}

In closed string field theory the algebraic structure includes a bilinear form $\langle\cdot, \cdot\rangle$ which satisfies $\langle A, B\rangle=(-1)^{(A+1)(B+1)}\langle B, A\rangle$, a BRST operator $Q$, and a series of graded-commutative string products $\left[B_{1}, \ldots, B_{m}\right]$ with $m \geq 2$ that satisfy a set of identities discussed in great detail in [6] and reviewed in [9]. The closed string field $\Psi$ is a ghost number two state of the matter plus ghosts conformal field theory, and the (infinitesimal) gauge parameter $\Xi$ is a state of ghost number one. The action in bosonic closed string field theory is

$$
S=-\frac{2}{\alpha^{\prime}}\left(\frac{1}{2}\langle\Psi, Q \Psi\rangle+\sum_{n=3}^{\infty} \frac{\kappa^{n-2}}{n !}\left\langle\Psi,\left[\Psi^{n-1}\right]\right\rangle\right),
$$

where $\kappa$ is the gravitational constant and we have rescaled $\Psi$ so that the kinetic term has no factor of $1 / \kappa^{2}$. The gauge transformation of bosonic closed string field theory is given by

$$
\delta_{\Xi} \Psi=Q \Xi+\sum_{n=1}^{\infty} \frac{\kappa^{n}}{n !}\left[\Psi^{n}, \Xi\right]
$$

and the equation of motion of the string field is

$$
\mathcal{F}(\Psi) \equiv Q \Psi+\sum_{n=2}^{\infty} \frac{\kappa^{n-1}}{n !}\left[\Psi^{n}\right]=0 .
$$


It is useful to examine the closed string field theory that arises when we expand the string field around a nonvanishing string field $\Psi_{Q}$. To do this, we let $\Psi \rightarrow \Psi_{Q}+\Psi$ in the string field theory action and reorganize the result in powers of $\Psi$. This was done in [6] where a new BRST-like operator $Q^{\prime}$ and new products $[\ldots]^{\prime}$ were introduced:

$$
\begin{gathered}
Q^{\prime} A \equiv Q A+\sum_{n=1}^{\infty} \frac{\kappa^{n}}{n !}\left[\Psi_{Q}^{n}, A\right] \\
{\left[B_{1}, B_{2}, \ldots, B_{m}\right]^{\prime} \equiv \sum_{n=0}^{\infty} \frac{\kappa^{n}}{n !}\left[\Psi_{Q}^{n}, B_{1}, B_{2}, \ldots, B_{m}\right] .}
\end{gathered}
$$

The operator $Q^{\prime}$ satisfies

$$
\left\langle Q^{\prime} A, B\right\rangle=(-1)^{A}\left\langle A, Q^{\prime} B\right\rangle .
$$

It is also natural to introduce graded-commutative shifted multilinear forms:

$$
\left\{B_{1}, B_{2}, \ldots, B_{n}\right\}^{\prime}=\left\langle B_{1},\left[B_{2}, \ldots, B_{n}\right]^{\prime}\right\rangle .
$$

As shown in equation (4.117) of [6],

$$
Q^{\prime}\left(Q^{\prime} A\right)=-\kappa\left[\mathcal{F}\left(\Psi_{Q}\right), A\right]^{\prime} .
$$

In subsection 3.2 we define the string field $\Psi_{Q}$ explicitly and show that it satisfies the equation of motion $\mathcal{F}\left(\Psi_{Q}\right)=0$. As a result, $Q^{\prime}$ squares to zero. Moreover, $Q^{\prime}$ and the new products also satisfy the main identity that the original BRST operator and products do. For example, $Q^{\prime}$ is a derivation of the primed product with two inputs:

$$
Q^{\prime}\left[B_{1}, B_{2}\right]^{\prime}=-\left[Q^{\prime} B_{1}, B_{2}\right]^{\prime}-(-1)^{B_{1}}\left[B_{1}, Q^{\prime} B_{2}\right]^{\prime} .
$$

Just as $Q$ fails to be a derivation of $[\cdot, \cdot, \cdot], Q^{\prime}$ fails to be a derivation of $[\cdot, \cdot, \cdot]^{\prime}$ :

$$
\begin{aligned}
0= & Q^{\prime}\left[B_{1}, B_{2}, B_{3}\right]^{\prime}+\left[Q^{\prime} B_{1}, B_{2}, B_{3}\right]^{\prime}+(-1)^{B_{1}}\left[B_{1}, Q^{\prime} B_{2}, B_{3}\right]^{\prime}+(-1)^{B_{1}+B_{2}}\left[B_{1}, B_{2}, Q^{\prime} B_{3}\right]^{\prime} \\
& +(-1)^{B_{1}}\left[B_{1},\left[B_{2}, B_{3}\right]^{\prime}\right]^{\prime}+(-1)^{B_{2}\left(1+B_{1}\right)}\left[B_{2},\left[B_{1}, B_{3}\right]^{\prime}\right]^{\prime}+\left[\left[B_{1}, B_{2}\right]^{\prime}, B_{3}\right]^{\prime} .
\end{aligned}
$$

Derivations of all of the string products play an important role in our construction of heterotic string field theory. An operation $X$ is a derivation if $X$ satisfies

$$
\begin{aligned}
X\left[B_{1}, B_{2}, \ldots, B_{m}\right]= & (-1)^{X}\left[X B_{1}, B_{2}, \ldots, B_{m}\right]+(-1)^{X\left(1+B_{1}\right)}\left[B_{1}, X B_{2}, \ldots, B_{m}\right] \\
& +\cdots+(-1)^{X\left(1+B_{1}+B_{2}+\cdots+B_{m-1}\right)}\left[B_{1}, B_{2}, \ldots, X B_{m}\right]
\end{aligned}
$$

for $m \geq 2$. In this paper we only consider derivations that further satisfy $X Q=(-1)^{X} Q X$. In fact, the graded-commutativity of $X$ with $Q$ is the statement that (3.15) holds for $m=1$ because the one-input product is defined by $[B] \equiv Q B\left[\underline{6}\right.$. While $Q$ and $X$ commute (or anticommute), $Q^{\prime}$ and $X$ do not. It is easy to show that for arbitrary string field $B$

$$
Q^{\prime}(X B)-(-1)^{X} X\left(Q^{\prime} B\right)=-\kappa\left[X \Psi_{Q}, B\right]^{\prime} .
$$




\subsection{Pure-gauge closed string fields}

Let us consider a 'large' pure-gauge closed string field $G(V)$ associated with a finite gauge parameter $V$. Such pure-gauge field can be built by successive infinitesimal gauge transformations but, as in any nonAbelian theory, the result depends on the path connecting 0 and $V$. The pure-gauge open string field $e^{-\Phi}\left(Q e^{\Phi}\right)$ corresponds to choosing a straight line connecting 0 and $\Phi$. For closed strings we also choose a straight line connecting 0 and $V$ and parametrize the path as $\tau V$ with $0 \leq \tau \leq 1$. Intermediate pure-gauge fields are given by $G(\tau V)$, and the final pure-gauge string field $G(V)$ corresponds to $G(\tau V)$ at $\tau=1$. This string field is defined by the following two conditions. First, $G(\tau V)$ vanishes at $\tau=0$, and second, $G(\tau V+d \tau V)$ and $G(\tau V)$ differ by a gauge transformation with gauge parameter $d \tau V$ :

$$
G(\tau V+d \tau V)-G(\tau V)=Q(d \tau V)+\sum_{n=1}^{\infty} \frac{\kappa^{n}}{n !}\left[G(\tau V)^{n}, d \tau V\right]+\mathcal{O}\left(d \tau^{2}\right) .
$$

Therefore, $G(\tau V)$ satisfies the differential equation

$$
\partial_{\tau} G(\tau V)=Q V+\sum_{n=1}^{\infty} \frac{\kappa^{n}}{n !}\left[G(\tau V)^{n}, V\right] \equiv Q_{G}^{\prime} V,
$$

with the initial condition $G(0)=0$.

It is in fact straightforward to solve (3.18) in an expansion in powers of $\kappa$. Let us expand the equation and $G$ as

$$
\partial_{\tau} G=Q V+\kappa[G, V]+\frac{\kappa^{2}}{2}[G, G, V]+\mathcal{O}\left(\kappa^{3}\right)
$$

and

$$
G=G^{(0)}+\kappa G^{(1)}+\kappa^{2} G^{(2)}+\mathcal{O}\left(\kappa^{3}\right) .
$$

The initial condition gives $G^{(n)}=0$ at $\tau=0$ for all $n \geq 0$. The equation at $\mathcal{O}\left(\kappa^{0}\right)$ is given by $\partial_{\tau} G^{(0)}=Q V$ and is solved by $G^{(0)}=\tau Q V$. The equation at $\mathcal{O}(\kappa)$ is $\partial_{\tau} G^{(1)}=\left[G^{(0)}, V\right]=[\tau Q V, V]$ and is solved by $G^{(1)}=\frac{\tau^{2}}{2}[Q V, V]$. It is straightforward to proceed to higher orders in this way. After setting $\tau=1$, the first few terms of $G(V)$ are given by

$$
G(V)=Q V+\frac{\kappa}{2}[V, Q V]+\frac{\kappa^{2}}{3 !}[V, Q V, Q V]+\frac{\kappa^{2}}{3 !}[V,[V, Q V]]+\mathcal{O}\left(\kappa^{3}\right) .
$$

Large gauge transformations in closed string field theory were considered long ago by Schubert 12. The class of terms that appear are all those constructed with $V$ and $Q V$ that are consistent with ghost number 9], and simple rules to determine the numerical coefficient of a given term were stated in [12]. Our result (3.21) is in agreement with the rules in [12].

Since $G(V)$ is a pure gauge, we expect it to satisfy the closed string field theory equation of motion. This is readily proven by evaluating $\partial_{\tau} \mathcal{F}(G(\tau V))$. Using (3.18) and (3.12) we find

$$
\begin{aligned}
\partial_{\tau} \mathcal{F}(G(\tau V)) & =\partial_{\tau}\left(Q G(\tau V)+\sum_{n=2}^{\infty} \frac{\kappa^{n-1}}{n !}\left[G(\tau V)^{n}\right]\right) \\
& =Q\left(\partial_{\tau} G(\tau V)\right)+\sum_{n=1}^{\infty} \frac{\kappa^{n}}{n !}\left[G(\tau V)^{n}, \partial_{\tau} G(\tau V)\right] \\
& =Q_{G}^{\prime}\left(\partial_{\tau} G(\tau V)\right)=Q_{G}^{\prime}\left(Q_{G}^{\prime} V\right)=-\kappa[\mathcal{F}(G(\tau V)), V]_{G}^{\prime},
\end{aligned}
$$


where a primed product with subscript $G$ is defined by (3.9) with $\Psi_{Q}$ replaced by $G(\tau V)$. We therefore have the following differential equation for $\mathcal{F}(G(\tau V))$ :

$$
\partial_{\tau} \mathcal{F}(G(\tau V))=\kappa[V, \mathcal{F}(G(\tau V))]_{G}^{\prime} .
$$

Since $G(\tau V)$ vanishes at $\tau=0, \mathcal{F}(G(0))=0$. The above first-order differential equation is solved by $\mathcal{F}(G(\tau V))=0$ for any $\tau$ so, by uniqueness, this is the solution with the given initial condition. After setting $\tau=1$, we thus obtain that

$$
\mathcal{F}(G(V))=Q G(V)+\sum_{n=2}^{\infty} \frac{\kappa^{n-1}}{n !}\left[G(V)^{n}\right]=0 .
$$

In open superstring field theory $A_{Q}$ is a pure-gauge open string field for each value of $t$. To define an analogous closed string field we proceed as follows. For any given string field $V$ we introduce a $t$-dependent string field $V(t)$, with $t \in[0,1]$, that connects the zero string field $0=V(t=0)$ and $V=V(t=1)$. We now define $\Psi_{Q}$ by

$$
\Psi_{Q} \equiv G(V(t))
$$

The string field $\Psi_{Q}$ is a pure-gauge closed string field for each value of $t$. Since $\Psi_{Q}$ satisfies the string field equation of motion, the operator

$$
Q^{\prime} \equiv Q_{\Psi_{Q}}^{\prime}
$$

and the primed products satisfy the familiar identities of closed string field theory. In particular, $Q^{\prime 2}$ vanishes.

\subsection{Derivations and associated string fields}

Let us next construct $\Psi_{t}, \Psi_{\delta}$, and $\Psi_{\eta}$ satisfying (3.1), (3.2), and (3.3). As in open superstring field theory, the existence of $\Psi_{\delta}$ is guaranteed because $\Psi_{Q}$ is a pure-gauge string field and therefore any infinitesimal variation of $\Psi_{Q}$ induced by a variation $\delta V(t)$ can be written as a gauge transformation $Q^{\prime} \Psi_{\delta}$ for some $\Psi_{\delta}$. The existence of $\Psi_{t}$ and $\Psi_{\eta}$ also follows since derivations can be viewed as formal variations.

We claim that the problem of explicit construction of these string fields reduces to that of constructing a string field $H(V, X V)$ which satisfies

$$
X G(V)=(-1)^{X} Q_{G}^{\prime} H(V, X V),
$$

where $X$ is any derivation of all of the closed string products and satisfies $[X, Q\}=0$. We view equation (3.27) as a relation between maps $V \rightarrow G(V)$ and $(V, X V) \rightarrow H(V, X V)$ that holds for arbitrary $V$ on which the action of $X$ is well defined.

Let us now demonstrate that (3.27) provides a construction of $\Psi_{t}, \Psi_{\delta}$, and $\Psi_{\eta}$. First note that equation (3.27) holds for any fixed $t$ if we replace each $V$ by $V(t)$. Since $\Psi_{Q}=G(V(t))$ and we write $Q^{\prime}=Q_{\Psi_{Q}}^{\prime}$, we have

$$
X \Psi_{Q}=(-1)^{X} Q^{\prime} H(V(t), X V(t)) .
$$


Since $\delta$ and $\eta$ are derivations that act with fixed $t$, the above equation clearly holds for $X=\delta$ and $X=\eta$. We thus find that $\Psi_{\delta}$ and $\Psi_{\eta}$ satisfying (3.2) and (3.3) are given by $\Psi_{\delta}=H(V(t), \delta V(t))$ and $\Psi_{\eta}=H(V(t), \eta V(t))$. In fact, equation (3.28) also holds for $X=\partial_{t}$. By construction, $\Psi_{Q}$ depends on $t$ only through $V(t)$ so $\partial_{t} \Psi_{Q}=\delta \Psi_{Q}$ with $\delta V(t)=\partial_{t} V(t)$. Since $\delta \Psi_{Q}=Q^{\prime} \Psi_{\delta}=Q^{\prime} H(V(t), \delta V(t)), \Psi_{t}$ satisfying (3.1) is given by $H\left(V(t), \partial_{t} V(t)\right)$. In summary,

$$
\begin{aligned}
\Psi_{t} & =H\left(V(t), \partial_{t} V(t)\right), \\
\Psi_{\delta} & =H(V(t), \delta V(t)), \\
\Psi_{\eta} & =H(V(t), \eta V(t)) .
\end{aligned}
$$

Equation (3.28) and the equations above are summarized as

$$
X \Psi_{Q}=(-1)^{X} Q^{\prime} \Psi_{X}, \quad \text { with } \quad \Psi_{X}=H(V(t), X V(t)),
$$

for $X=\partial_{t}, \delta$, and $\eta$.

In open string field theory, the string field $A_{X}$ that satisfies $X A_{Q}=(-1)^{X} Q^{\prime} A_{X}$ is obtained by replacing $Q$ by $X$ in the expression for $A_{Q}$. In the case of closed string field theory, however, the construction of $H(V, X V)$ or, equivalently, $\Psi_{X}$ is more complicated. As in the case of $G(V)$, we construct $H(V, X V)$ as the solution to a differential equation for $H(V, X V ; \tau)$, evaluated at $\tau=1$. To derive the requisite differential equation we define the auxiliary string field $\mathcal{H}(\tau)$ inspired by the $\tau$-dependent version of (3.27):

$$
\mathcal{H}(\tau) \equiv Q_{G}^{\prime} H(V, X V ; \tau)-(-1)^{X} X G(\tau V) .
$$

Equation (3.27), which we want to prove, is equivalent to $\mathcal{H}(1)=0$. We compute $\partial_{\tau} \mathcal{H}$ using (3.16) and (3.18). The result is

$$
\partial_{\tau} \mathcal{H}=\kappa[V, \mathcal{H}]_{G}^{\prime}+Q_{G}^{\prime}\left(\partial_{\tau} H(V, X V ; \tau)-X V-\kappa[V, H(V, X V ; \tau)]_{G}^{\prime}\right) .
$$

We will demand that the expression in parentheses on the right-hand side vanishes:

$$
\partial_{\tau} H(V, X V ; \tau)=X V+\kappa[V, H(V, X V ; \tau)]_{G}^{\prime} .
$$

With this differential equation for $H(V, X V ; \tau)$, the differential equation for $\mathcal{H}$ becomes

$$
\partial_{\tau} \mathcal{H}=\kappa[V, \mathcal{H}]_{G}^{\prime} .
$$

With initial condition $\mathcal{H}(\tau=0)=0$, this differential equation ensures that $\mathcal{H}$ vanishes for any $\tau$, and (3.27) is proven. We must therefore solve (3.35) with initial conditions that ensure that $\mathcal{H}(\tau=0)=0$, namely,

$$
Q_{G}^{\prime} H(V, X V ; \tau)=(-1)^{X} X G(\tau V) \quad \text { at } \quad \tau=0 .
$$

Note that $X G(\tau V)$ vanishes at $\tau=0$ because $X$ does not act on the auxiliary parameter $\tau$ and $G(\tau V)$ vanishes for $\tau=0$ for any finite $V$. We can then require

$$
H(V, X V ; \tau=0)=0
$$


and (3.37) holds with both terms equal to zero. As in the case of the differential equation for $G(\tau V)$, it is straightforward to solve (3.35) in powers of $\kappa$. With the initial condition (3.38) we find that the first few terms in $H(V, X V)$ are given by

$$
H(V, X V)=X V+\frac{\kappa}{2}[V, X V]+\frac{\kappa^{2}}{3}[V, Q V, X V]+\frac{\kappa^{2}}{3 !}[V,[V, X V]]+\mathcal{O}\left(\kappa^{3}\right) .
$$

This result can be used to write explicit expressions for $\Psi_{t}, \Psi_{\delta}$, and $\Psi_{\eta}$ using (3.29), (3.30), and (3.31).

\subsection{Heterotic string field theory action}

Having constructed $\Psi_{Q}, \Psi_{t}, \Psi_{\delta}$, and $\Psi_{\eta}$ satisfying (3.1), (3.2), and (3.3), we now show that the action (3.4) has the requisite gauge invariances. The proof is completely parallel to the one for open superstring field theory.

In the computation of $\delta S$ we will use the identity:

$$
Q^{\prime}\left(\partial_{t} \Psi_{\delta}-\delta \Psi_{t}-\kappa\left[\Psi_{t}, \Psi_{\delta}\right]^{\prime}\right)=0 .
$$

This identity, which is the closed string analog of (2.20), follows from (3.1) and (3.2) with the help of (3.16):

$$
\begin{aligned}
0 & =\partial_{t}\left(Q^{\prime} \Psi_{\delta}\right)-\delta\left(Q^{\prime} \Psi_{t}\right) \\
& =Q^{\prime}\left(\partial_{t} \Psi_{\delta}\right)+\kappa\left[\partial_{t} \Psi_{Q}, \Psi_{\delta}\right]^{\prime}-Q^{\prime}\left(\delta \Psi_{t}\right)-\kappa\left[\delta \Psi_{Q}, \Psi_{t}\right]^{\prime} \\
& =Q^{\prime}\left(\partial_{t} \Psi_{\delta}-\delta \Psi_{t}-\kappa\left[\Psi_{t}, \Psi_{\delta}\right]^{\prime}\right) .
\end{aligned}
$$

Let us compute the variation of the integrand of the action:

$$
\delta\left\langle\eta \Psi_{t}, \Psi_{Q}\right\rangle=\left\langle\eta \delta \Psi_{t}, \Psi_{Q}\right\rangle+\left\langle\eta \Psi_{t}, \delta \Psi_{Q}\right\rangle
$$

The first term on the right-hand side is evaluated as follows:

$$
\begin{aligned}
\left\langle\eta \delta \Psi_{t}, \Psi_{Q}\right\rangle & =-\left\langle\delta \Psi_{t}, \eta \Psi_{Q}\right\rangle=\left\langle\delta \Psi_{t}, Q^{\prime} \Psi_{\eta}\right\rangle=-\left\langle Q^{\prime} \delta \Psi_{t}, \Psi_{\eta}\right\rangle \\
& =-\left\langle Q^{\prime}\left(\partial_{t} \Psi_{\delta}+\kappa\left[\Psi_{\delta}, \Psi_{t}\right]^{\prime}\right), \Psi_{\eta}\right\rangle \\
& =-\left\langle\partial_{t} \Psi_{\delta}+\kappa\left[\Psi_{\delta}, \Psi_{t}\right]^{\prime}, \eta \Psi_{Q}\right\rangle \\
& =\left\langle\eta \partial_{t} \Psi_{\delta}, \Psi_{Q}\right\rangle-\kappa\left\langle\eta \Psi_{Q},\left[\Psi_{\delta}, \Psi_{t}\right]^{\prime}\right\rangle \\
& =\left\langle\eta \partial_{t} \Psi_{\delta}, \Psi_{Q}\right\rangle-\kappa\left\{\eta \Psi_{Q}, \Psi_{\delta}, \Psi_{t}\right\}^{\prime}
\end{aligned}
$$

The second term on the right-hand side is evaluated as follows:

$$
\begin{aligned}
\left\langle\eta \Psi_{t}, \delta \Psi_{Q}\right\rangle & =\left\langle\eta \Psi_{t}, Q^{\prime} \Psi_{\delta}\right\rangle=\left\langle Q^{\prime}\left(\eta \Psi_{t}\right), \Psi_{\delta}\right\rangle \\
& =-\left\langle\eta\left(Q^{\prime} \Psi_{t}\right), \Psi_{\delta}\right\rangle-\kappa\left\langle\left[\eta \Psi_{Q}, \Psi_{t}\right]^{\prime}, \Psi_{\delta}\right\rangle \\
& =-\left\langle\eta \partial_{t} \Psi_{Q}, \Psi_{\delta}\right\rangle-\kappa\left\langle\left[\eta \Psi_{Q}, \Psi_{t}\right]^{\prime}, \Psi_{\delta}\right\rangle \\
& =\left\langle\eta \Psi_{\delta}, \partial_{t} \Psi_{Q}\right\rangle-\kappa\left\langle\Psi_{\delta},\left[\eta \Psi_{Q}, \Psi_{t}\right]^{\prime}\right\rangle \\
& =\left\langle\eta \Psi_{\delta}, \partial_{t} \Psi_{Q}\right\rangle+\kappa\left\{\eta \Psi_{Q}, \Psi_{\delta}, \Psi_{t}\right\}^{\prime} .
\end{aligned}
$$


Therefore, the variation of the integrand of the action is given by

$$
\delta\left\langle\eta \Psi_{t}, \Psi_{Q}\right\rangle=\partial_{t}\left\langle\eta \Psi_{\delta}, \Psi_{Q}\right\rangle
$$

Since this variation is a total $t$ derivative, the variation of the action is given by

$$
\delta S=\frac{2}{\alpha^{\prime}}\left\langle\eta \bar{\Psi}_{\delta}, \bar{\Psi}_{Q}\right\rangle=-\frac{2}{\alpha^{\prime}}\left\langle\bar{\Psi}_{\delta}, \eta \bar{\Psi}_{Q}\right\rangle,
$$

where the bar, as before, denotes evaluation at $t=1$. Using (3.3), the variation of the action can also be written as

$$
\delta S=\frac{2}{\alpha^{\prime}}\left\langle\bar{\Psi}_{\delta},{\overline{Q^{\prime}}}^{\prime} \bar{\Psi}_{\eta}\right\rangle=-\frac{2}{\alpha^{\prime}}\left\langle\bar{Q}^{\prime} \bar{\Psi}_{\delta}, \bar{\Psi}_{\eta}\right\rangle .
$$

Recall that $\Psi_{\delta}$ depends on $t$ only through $V(t)$ and $\delta V(t)$. The first few terms of $\Psi_{\delta}$ as a series in powers of $\kappa$ are

$$
\Psi_{\delta}=\delta V(t)+\frac{\kappa}{2}[V(t), \delta V(t)]+\frac{\kappa^{2}}{3}[V(t), Q V(t), \delta V(t)]+\frac{\kappa^{2}}{3 !}[V(t),[V(t), \delta V(t)]]+\mathcal{O}\left(\kappa^{3}\right) .
$$

It is straightforward to verify that $\Psi_{\delta}$ is an invertible function of $\delta V(t)$, at least in the expansion in powers of $\kappa$. One writes $\delta V(t)=v^{(0)}+\kappa v^{(1)}+\kappa^{2} v^{(2)}+\ldots$, substitutes in (3.48), and solves recursively for $v^{(0)}, v^{(1)}, v^{(2)}, \ldots$, without encountering obstructions. The first few terms of the resulting expression are

$$
\delta V(t)=\Psi_{\delta}+\frac{\kappa}{2}\left[\Psi_{\delta}, V(t)\right]+\frac{\kappa^{2}}{3}\left[\Psi_{\delta}, Q V(t), V(t)\right]+\frac{\kappa^{2}}{12}\left[\left[\Psi_{\delta}, V(t)\right], V(t)\right]+\mathcal{O}\left(\kappa^{3}\right) .
$$

Since the relation between $\Psi_{\delta}$ and $\delta V(t)$ is invertible, arbitrary $\delta V$ is equivalent to arbitrary $\bar{\Psi}_{\delta}$. We therefore conclude that (3.46) gives the following equation of motion:

$$
\eta \bar{\Psi}_{Q}=0
$$

The equation of motion can also be written as $\overline{Q^{\prime}} \bar{\Psi}_{\eta}=0$, as is manifest from the expression (3.47).

Let us now consider the gauge invariances of the action. The action is manifestly invariant under a variation for which $\bar{\Psi}_{\delta}=\eta \Omega$ :

$$
\delta_{\Omega} S=\frac{2}{\alpha^{\prime}}\left\langle\eta(\eta \Omega), \bar{\Psi}_{Q}\right\rangle=0 .
$$

We therefore have a gauge transformation

$$
\Psi_{\delta}=\eta \Omega(t),
$$

where $\Omega(0)=0$ and $\Omega(1)=\Omega$. The formula (3.49) can be used to find, if desired, an explicit expression for the gauge variation $\delta_{\Omega} V(t)$ associated with (3.52). We immediately find that $\delta_{\Omega} V=\delta_{\Omega} V(t=1)$ is given by

$$
\delta_{\Omega} V=\eta \Omega+\frac{\kappa}{2}[\eta \Omega, V]+\frac{\kappa^{2}}{3}[\eta \Omega, Q V, V]+\frac{\kappa^{2}}{12}[[\eta \Omega, V], V]+\mathcal{O}\left(\kappa^{3}\right)
$$


By construction, the gauge parameter $\Omega$ always appears in the form $\eta \Omega$. This was also the requirement used in [9] to fix the ambiguity from field redefinition of the gauge parameter. Therefore $\delta_{\Omega} V$ in (3.53) should coincide with that in [9. We in fact found that this is the case up to the order computed in 9].

The action is also invariant under a variation for which $\bar{\Psi}_{\delta}=\overline{Q^{\prime}} \Lambda^{\prime}$ :

$$
\delta_{\Lambda^{\prime}} S=-\frac{2}{\alpha^{\prime}}\left\langle\overline{Q^{\prime}}\left(\overline{Q^{\prime}} \Lambda^{\prime}\right), \bar{\Psi}_{\eta}\right\rangle=0 .
$$

We therefore have the gauge transformation

$$
\Psi_{\delta}=Q^{\prime} \Lambda^{\prime}(t)
$$

where $\Lambda^{\prime}(0)=0$ and $\Lambda^{\prime}(1)=\Lambda^{\prime}$. A formula for the associated gauge variation $\delta_{\Lambda^{\prime}} V$ can be obtained using (3.49) with $\bar{\Psi}_{\delta}=\overline{Q^{\prime}} \Lambda^{\prime}$. When we expand this formula in powers of $\kappa, \Lambda^{\prime}$ sometimes appears in the form $Q \Lambda^{\prime}$, but sometimes it appears without $Q$. The gauge transformation $\delta_{\Lambda} V$ in [9] was fixed by the requirement that the gauge parameter $\Lambda$ always appear in the form $Q \Lambda$. The transformations do not coincide exactly, but they should be related by redefinition of the gauge parameters. We in fact find

$$
\Lambda^{\prime}=\Lambda+\kappa[V, \Lambda]+\frac{\kappa^{2}}{2}[V, Q V, \Lambda]+\frac{\kappa^{2}}{2}[V,[V, \Lambda]]+\mathcal{O}\left(\kappa^{3}\right) .
$$

\subsection{Reparameterization invariance and linear homotopy}

We have shown that the heterotic string field theory action (3.4) is invariant under the gauge transformations $\delta_{\Lambda^{\prime}} V$ and $\delta_{\Omega} V$. Moreover, the action is independent of the parametrized path $V(t)$ in field space that joins $V(0)=0$ to $V(1)=V$. We established this fact by showing that the variation of the action depends only on the values of the variation and fields at $t=1$. To write the string field theory action we can therefore choose any path $V(t)$ from 0 to $V$. The path independence implies that the action is invariant under reparametrization of any chosen path. While the independence of the parametrized path is not manifest in the action, this reparametrization invariance is manifest. We first note that $V(t)$ is scalar under the reparametrizations: $V(t)=V^{\prime}\left(t^{\prime}\right)$. Moreover, $\Psi_{Q}$ and $\Psi_{t}$ transform as

$$
\Psi_{Q}(V(t))=\Psi_{Q}^{\prime}\left(V^{\prime}\left(t^{\prime}\right)\right), \quad \Psi_{t}(V(t))=\frac{d t^{\prime}}{d t} \Psi_{t}^{\prime}\left(V^{\prime}\left(t^{\prime}\right)\right)
$$

The field $\Psi_{Q}$ is a scalar because it depends on $t$ only through $V(t)$. The transformation of $\Psi_{t}$ follows from (3.1) and the fact that $Q^{\prime}$ is a scalar. The two equations in (3.57) imply the reparameterization invariance of the action (3.4).

The action takes a simple form when we choose a straight path from 0 to $V$ and we parameterize it linearly: $V(t)=t V$. In this case $\Psi_{Q}=G(t V)$ from the definition (3.25) and $\partial_{t} \Psi_{Q}=\partial_{t} G(t V)=Q^{\prime} V$ using (3.18). Comparing with (3.1) we conclude that $\Psi_{t}=V$, and the action (3.4) becomes

$$
S=\frac{2}{\alpha^{\prime}} \int_{0}^{1} d t\langle\eta V, G(t V)\rangle .
$$


This form of the action is very useful. The coefficient of any term in $\Psi_{Q}=G(t V)$ can be easily obtained from Schubert's rules [12], and the integral over $t$ is trivial. Using the expression for $G(t V)$ as a series in $\kappa$, the first few terms of the action are given by

$$
S=\frac{2}{\alpha^{\prime}} \int_{0}^{1} d t\left\langle\eta V, t Q V+\frac{\kappa}{2} t^{2}[V, Q V]+\ldots\right\rangle=\frac{2}{\alpha^{\prime}}\left(\frac{1}{2}\langle\eta V, Q V\rangle+\frac{\kappa}{3 !}\langle\eta V,[V, Q V]\rangle+\ldots\right) .
$$

These terms agree with those in the action given in $[9$. In fact, one can easily check the agreement for all the terms that were determined in 9 .

\section{Heterotic string action in standard WZW form}

We have seen that the familiar WZW bosonic action (2.3), which involves a two-dimensional and a three-dimensional term, can be written in the less familiar compact form (2.4). A similar fact is true for open superstrings: the original WZW action (2.7) can be written as in (2.15). We would like to know if the heterotic string action

$$
S=\frac{2}{\alpha^{\prime}} \int_{0}^{1} d t\left\langle\eta \Psi_{t}, \Psi_{Q}\right\rangle
$$

can be transformed into the more familiar WZW form. We find that such a familiar form is obtained when $V(t)=t V$, but there is an additional term for general paths $V(t)$.

We begin by a straightforward attempt to transform the action (4.1). Our experience in performing a similar transformation for open superstrings indicates that in addition to (3.1) and (3.3), we must use the closed string analog of equation (2.31), which states that the field strength $F_{\eta t}$ vanishes. Nevertheless, for closed strings we find that the following combination $E_{\eta t}$ of operators does not vanish:

$$
E_{\eta t} \equiv \eta \Psi_{t}-\partial_{t} \Psi_{\eta}+\kappa\left[\Psi_{\eta}, \Psi_{t}\right]^{\prime} \neq 0
$$

Indeed, $\Psi_{t}$ and $\Psi_{\eta}$ have already been defined, and a computation shows that $E_{\eta t}$ does not vanish for arbitrary $V(t)$. We proceed, anyway, by writing

$$
\begin{aligned}
S & =\frac{1}{\alpha^{\prime}} \int_{0}^{1} d t\left\langle\eta \Psi_{t}, \Psi_{Q}\right\rangle-\frac{1}{\alpha^{\prime}} \int_{0}^{1} d t\left\langle\Psi_{t}, \eta \Psi_{Q}\right\rangle \\
& =\frac{1}{\alpha^{\prime}} \int_{0}^{1} d t\left\langle\partial_{t} \Psi_{\eta}-\kappa\left[\Psi_{\eta}, \Psi_{t}\right]^{\prime}, \Psi_{Q}\right\rangle+\frac{1}{\alpha^{\prime}} \int_{0}^{1} d t\left\langle E_{\eta t}, \Psi_{Q}\right\rangle+\frac{1}{\alpha^{\prime}} \int_{0}^{1} d t\left\langle\Psi_{t}, Q^{\prime} \Psi_{\eta}\right\rangle
\end{aligned}
$$

A little reorganization gives

$$
S=\frac{1}{\alpha^{\prime}} \int_{0}^{1} d t\left(\left\langle\partial_{t} \Psi_{\eta}, \Psi_{Q}\right\rangle+\left\langle\Psi_{\eta}, Q^{\prime} \Psi_{t}\right\rangle\right)+\frac{\kappa}{\alpha^{\prime}} \int_{0}^{1} d t\left\langle\Psi_{Q},\left[\Psi_{\eta}, \Psi_{t}\right]^{\prime}\right\rangle+\frac{1}{\alpha^{\prime}} \int_{0}^{1} d t\left\langle E_{\eta t}, \Psi_{Q}\right\rangle .
$$

Using (3.1) and the graded commutativity of the multilinear forms, we have

$$
S=\frac{1}{\alpha^{\prime}} \int_{0}^{1} d t \partial_{t}\left\langle\Psi_{\eta}, \Psi_{Q}\right\rangle+\frac{\kappa}{\alpha^{\prime}} \int_{0}^{1} d t\left\langle\Psi_{t},\left[\Psi_{\eta}, \Psi_{Q}\right]^{\prime}\right\rangle+\frac{1}{\alpha^{\prime}} \int_{0}^{1} d t\left\langle E_{\eta t}, \Psi_{Q}\right\rangle .
$$


Integrating the first term we find

$$
S=\frac{1}{\alpha^{\prime}}\left(\left\langle\bar{\Psi}_{\eta}, \bar{\Psi}_{Q}\right\rangle+\kappa \int_{0}^{1} d t\left\langle\Psi_{t},\left[\Psi_{\eta}, \Psi_{Q}\right]^{\prime}\right\rangle+\int_{0}^{1} d t\left\langle E_{\eta t}, \Psi_{Q}\right\rangle\right) .
$$

The last term in the action is reparameterization invariant and is necessary for the equivalence with (4.1). The above action is unusual but represents some kind of generalized WZW form. In ordinary WZW theory, the connections are flat and all field strengths vanish. The open string analog of $E_{\eta t}$ is the vanishing field strength $F_{\eta t}$.

We now prove that $E_{\eta t}$ vanishes when we choose a linear path $V(t)=t V$. We have shown in the lines above equation (3.58) that in this case $\Psi_{t}=V$. So we would like to show that

$$
\eta V-\partial_{t} \Psi_{\eta}+\kappa\left[\Psi_{\eta}, V\right]^{\prime}=0
$$

Consider equation (3.35) with $\tau$ replaced by $t$ :

$$
\partial_{t} H(V, X V ; t)=X V+\kappa[V, H(V, X V ; t)]^{\prime}
$$

It is straightforward to show from (3.35) that the solution $H$ satisfies the rescaling property

$$
H(V, X V ; \tau)=H\left(\frac{1}{a} V, \frac{1}{a} X V ; a \tau\right)
$$

for any constant $a$. Using this rescaling, equation (4.8) becomes

$$
\partial_{t} H(t V, X t V ; 1)=X V+\kappa[V, H(t V, X t V ; 1)]^{\prime} .
$$

Since $t V=V(t)$, and the $H$ functions are evaluated at $t=1$, we have

$$
\partial_{t} \Psi_{X}=X V+\kappa\left[V, \Psi_{X}\right]^{\prime}
$$

For $X=\eta$, this equation is equivalent to (4.7), which is what we wanted to establish. As a result

$$
S=\frac{1}{\alpha^{\prime}}\left(\left\langle\bar{\Psi}_{\eta}, \bar{\Psi}_{Q}\right\rangle+\kappa \int_{0}^{1} d t\left\langle\Psi_{t},\left[\Psi_{\eta}, \Psi_{Q}\right]^{\prime}\right\rangle\right), \quad V(t)=t V .
$$

This is our closest analog of the familiar WZW form. Note that the action is formally cubic in the 'connections' $\Psi_{Q}, \Psi_{t}$, and $\Psi_{\eta}$. The shifted product $[\cdot, \cdot]^{\prime}$ contains additional dependence on $\Psi_{Q}$, but the above expression and our earlier analysis show that it is a natural ingredient. Some of the nonpolynomiality of closed string field theory appears in the construction of $\Psi_{Q}, \Psi_{t}$, and $\Psi_{\eta}$, and further nonpolynomiality is subsumed in the shifted product. In open string field theory, there is no shifted product because the action is cubic.

To gain some understanding of the above complications, we discuss the definition of field strengths for closed strings. We explained right after equation (2.12) that the action of the shifted BRST operator $Q^{\prime}$ in open superstrings is that of a covariant derivative. We thus set

$$
\nabla_{Q}=Q^{\prime}
$$


In ordinary field theory the definition $F_{\mu \nu} \equiv\left[\nabla_{\mu}, \nabla_{\nu}\right]$ means that $F_{\mu \nu}$ is read from the equation $F_{\mu \nu} \cdot A=\nabla_{\mu}\left(\nabla_{\nu} A\right)-\nabla_{\nu}\left(\nabla_{\mu} A\right)$, valid for arbitrary $A$. For closed strings we use the two-input product to write

$$
\left[F_{Q Q}, A\right]^{\prime} \equiv\left\{\nabla_{Q}, \nabla_{Q}\right\} A=2 Q^{\prime}\left(Q^{\prime} A\right)
$$

Using (3.12) we find

$$
\left[F_{Q Q}, A\right]^{\prime}=-2 \kappa\left[\mathcal{F}\left(\Psi_{Q}\right), A\right]^{\prime} \quad \rightarrow \quad F_{Q Q}=-2 \kappa \mathcal{F}\left(\Psi_{Q}\right)
$$

As desired, the field strength $F_{Q Q}$ vanishes because $\Psi_{Q}$ satisfies the equation of motion. For derivations $X$ of all string products (that commute or anti-commute with $Q$ ) we introduce covariant derivatives as

$$
\nabla_{X} B \equiv X B-(-1)^{X} \kappa\left[\Psi_{X}, B\right]^{\prime}
$$

We can now try to define the field strengths that involve $Q$ and one derivation $X$ of all closed string products by $\left[F_{Q X}, A\right]^{\prime} \equiv\left[\nabla_{Q}, \nabla_{X}\right\} A$. A quick evaluation of the right hand side shows that the definition is consistent and

$$
F_{Q X}=-\kappa\left(X \Psi_{Q}-(-1)^{X} Q^{\prime} \Psi_{X}\right)
$$

As desired, the commutator of covariant derivatives has produced the quantity that we know vanishes. This construction furnishes vanishing field strengths $F_{Q \eta}$, and $F_{Q t}$, and $F_{Q \delta}$.

So far, our development has proceeded as well as it did for open strings. Consider now two derivations $X$ and $Y$ which commute or anticommute. We are naturally led to define a field strength $F_{X Y}$ by $\left[F_{X Y}, A\right]^{\prime} \equiv\left[\nabla_{X}, \nabla_{Y}\right\} A$. This time, however, an evaluation of the right-hand side shows that it is not of the form $[\cdots, A]^{\prime}$ because the Jacobi identity does not hold strictly in closed string theory (see (3.14) $)$. We thus fail to define the field strength $F_{X Y}$. It is nevertheless clear that a certain combination $E_{X Y}$ (that we cannot call a field strength) plays a significant role:

$$
E_{X Y} \equiv X \Psi_{Y}-(-1)^{X Y} Y \Psi_{X}-(-1)^{X} \kappa\left[\Psi_{X}, \Psi_{Y}\right]^{\prime} .
$$

The heterotic string action would take the standard WZW form if $E_{\eta t}$ vanished for arbitrary paths. A direct computation using the formulae for $\Psi_{X}$ and $\Psi_{Y}$ makes it clear that $E_{X Y}$ does not generally vanish: ${ }^{2}$

$$
E_{X Y}=-\frac{1}{3} \kappa^{2} Q^{\prime}[X V(t), V(t), Y V(t)]^{\prime}+\mathcal{O}\left(\kappa^{3}\right)
$$

One can use $F_{Q X}=0$ and $F_{Q Y}=0$ to verify that $Q^{\prime} E_{X Y}=0$. Indeed, $Q^{\prime} E_{\delta t}=0$ was used to prove the gauge invariance of the heterotic string theory. A general argument indicates that $Q^{\prime}$ has no cohomology ${ }^{3}$ so we expect $E_{X Y}=Q^{\prime} \Lambda_{X Y}$ for some $\Lambda_{X Y}$. It remains to be investigated if there exist

\footnotetext{
${ }^{2}$ It is a nontrivial question if one can make $E_{X Y}=0$ by choosing appropriate representatives for $\Psi_{X}$ and $\Psi_{Y}$ (recall $\Psi_{X} \sim \Psi_{X}+Q^{\prime} \Lambda_{X}$ and $\left.\Psi_{Y} \sim \Psi_{Y}+Q^{\prime} \Lambda_{Y}\right)$.

${ }^{3}$ In the large Hilbert space $\eta$ has trivial cohomology. Similarly, the BRST operator $Q$ has trivial cohomology. This follows from $\{Q, R\}=1$, where $R$ is the zero mode of $c \xi \partial \xi e^{-2 \phi}$. Any vertex operator $V$ satisfying $Q V=0$ can be written as $V=\{Q, R\} V=Q R V$, so $Q$ has trivial cohomology. We now use an expansion in powers of $\kappa$ to show that $Q^{\prime}$, which differs from $Q$ by terms with positive powers of $\kappa$, also has trivial cohomology. Consider a state $V=\sum_{n=0}^{\infty} \kappa^{n} V_{n}$. Then $Q^{\prime} V=0$ implies that $Q V_{0}=0$, and consequently $V_{0}=Q \Omega_{0}$. Therefore $\left(V-Q^{\prime} \Omega_{0}\right)=\sum_{n=1}^{\infty} \kappa^{n} W_{n}$ for some $W_{n}$ where $n \geq 1$. This time, $Q^{\prime}\left(V-Q^{\prime} \Omega_{0}\right)=0$ implies that $W_{1}=Q \Omega_{1}$, using a similar construction. Continuing this procedure forever, one finds $V=Q^{\prime}\left(\sum_{n=0}^{\infty} \Omega_{n}\right)$ for some $\Omega_{n}$. So $Q^{\prime}$ has trivial cohomology in the large Hilbert space.
} 
satisfactory definitions of field strengths in closed string theory.

\section{Conclusions}

In this paper we proposed the following action for the NS sector of heterotic string field theory:

$$
S=\frac{2}{\alpha^{\prime}} \int_{0}^{1} d t\left\langle\eta \Psi_{t}, \Psi_{Q}\right\rangle
$$

Here $\Psi_{Q}=G(V(t))$, where $G(V)=Q V+\ldots$ is a pure-gauge solution of the bosonic closed string field theory equation of motion. The action uses an NS string field $V(t)$ in the large Hilbert space, with $V(0)=0$ and $V(1)=V$. Because of its topological properties, the above action depends only on $V$. The string field $\Psi_{t}$ satisfies $\partial_{t} \Psi_{Q}=Q^{\prime} \Psi_{t}$, where $Q^{\prime}$ is the BRST operator around the background $\Psi_{Q}$.

The equation of motion implied by the action (5.1) is $\eta \bar{\Psi}_{Q}=0$, where the bar denotes evaluation at $t=1$. The action is invariant under nonlinear gauge transformations of the form $\delta V=Q \Lambda+$ $\eta \Omega+\ldots$ where the terms indicated by dots can be explicitly determined. This equation of motion and gauge invariance at linearized level reproduce the heterotic NS spectrum, and the gauge invariance is nonlinearly extended without using picture-changing operators. These two features convince us that (5.1) is correct. A particularly simple and useful form of the action is obtained by choosing $V(t)=t V$ :

$$
S=\frac{2}{\alpha^{\prime}} \int_{0}^{1} d t\langle\eta V, G(t V)\rangle \quad \text { when } \quad V(t)=t V .
$$

The action (5.1) is written as a WZW model in the sense that (1) gauge transformations generated by $Q$ and $\eta$ correspond to holomorphic and antiholomorphic gauge transformations, (2) the action uses pure-gauge fields, (3) the action is written using a third dimension, and (4) the action takes a form which has an analog in ordinary WZW theory. Since closed string products have properties altogether different from those of the open string star product, some features of our heterotic action are new. First, our action can be written in the standard WZW form only when the string field $V(t)$ depends on the third dimension $t$ of the WZW model as $V(t)=t V$ (see (4.12)). Second, the language of flat connections and vanishing field strengths, which works well in open superstring field theory (and ordinary WZW theory), does not seem totally appropriate for heterotic strings. Since the lowest closed string product $[\cdot, \cdot]$ does not satisfy the Jacobi identity, it does not seem possible to define field strengths by commutators of covariant derivatives that use the fields $\Psi_{\eta}$ and $\Psi_{t}$. Third, while in open superstrings $Q$ and $\eta$ are both derivations of the star algebra and are on the same footing, in heterotic string theory $\eta$ is a derivation of all closed string products but $Q$ is not. Consequently, $Q$ and $\eta$ are not on the same footing and the fields $\Psi_{Q}$ and $\Psi_{\eta}$ take inequivalent forms. It would be exciting to find a completely natural language for a geometrical formulation of the heterotic string action.

There are several possible generalizations of our results. Open superstring field theory generalizes to open $N=2$ string field theory [13] by replacing $Q$ and $\eta$ with the generators $G^{+}$and $\tilde{G}^{+}$from the $N=4$ topological description of the open $N=2$ string [14, 15]. Since the open bosonic string can be "embedded" in an open $N=2$ string [16], the WZW action for open superstring field theory can also 
be used to describe open bosonic string field theory. Similarly, our heterotic results should generalize to heterotic $N=2$ string field theory [17] by replacing $Q_{L}+Q_{R}$ and $\eta_{L}$ with the generators $G_{L}^{+}+Q_{R}$ and $\tilde{G}_{L}^{+}$from the $N=4$ topological description of the heterotic $N=2$ string. Since the closed bosonic string can be "embedded" in a heterotic $N=2$ string, our heterotic action may be used to describe closed bosonic string field theory.

One possible application of our action is the study of tachyon condensation. Just as the open superstring field theory action was useful for testing Sen's conjectures for open superstring tachyon condensation [18, it should be possible to use our heterotic string field theory action to test conjectures for closed superstring tachyon condensation [19].

Our results indicate a close and intriguing relationship between structures in open superstring field theory and heterotic string field theory. It is tempting to speculate that such relationships exist because of Type I/heterotic dualities. It would also be interesting to generalize our construction to describe the Ramond sector of heterotic string field theory. Finally, it is still unknown how to construct actions or equations of motion for Type II superstring field theory. Since this theory has an additional fermionic direction $\eta_{R}$, the construction might require new ingredients.

\section{Acknowledgments}

The work of Y.O. and B.Z. was supported in part by the DOE grant DF-FC02-94ER40818. N.B. would like to thank Warren Siegel for useful discussions, and CNPq grant 300256/94-9, Pronex grant 66.2002/1998-9, and FAPESP grant 99/12763-0 for partial financial support.

\section{References}

[1] E. Witten, "Noncommutative Geometry And String Field Theory," Nucl. Phys. B 268, 253 (1986).

[2] E. Witten, "Interacting Field Theory Of Open Superstrings," Nucl. Phys. B 276, 291 (1986).

[3] C. Wendt, "Scattering Amplitudes And Contact Interactions In Witten's Superstring Field Theory," Nucl. Phys. B 314, 209 (1989); C. R. Preitschopf, C. B. Thorn and S. A. Yost, "Superstring Field Theory," Nucl. Phys. B 337, 363 (1990); I. Y. Arefeva, P. B. Medvedev and A. P. Zubarev, "New Representation For String Field Solves The Consistence Problem For Open Superstring Field," Nucl. Phys. B 341, 464 (1990).

[4] D. Friedan, E. J. Martinec and S. H. Shenker, "Conformal Invariance, Supersymmetry And String Theory," Nucl. Phys. B 271, 93 (1986).

[5] N. Berkovits, "SuperPoincare invariant superstring field theory," Nucl. Phys. B 450, 90 (1995) [Erratumibid. B 459, 439 (1996)] arXiv:hep-th/9503099.

[6] B. Zwiebach, "Closed string field theory: Quantum action and the B-V master equation," Nucl. Phys. B 390, 33 (1993) arXiv:hep-th/9206084.

[7] M. Saadi and B. Zwiebach, "Closed String Field Theory From Polyhedra," Annals Phys. 192, 213 (1989); T. Kugo, H. Kunitomo and K. Suehiro, "Nonpolynomial Closed String Field Theory," Phys. Lett. B 226, 48 (1989); T. Kugo and K. Suehiro, "Nonpolynomial Closed String Field Theory: Action And Its Gauge Invariance," Nucl. Phys. B 337, 434 (1990); M. Kaku, "Geometric Derivation Of String Field Theory From First Principles: Closed Strings And Modular Invariance," Phys. Rev. D 38, 3052 (1988); M. Kaku and J. Lykken, "Modular Invariant Closed String Field Theory," Phys. Rev. D 38, 3067 (1988). 
[8] R. Saroja and A. Sen, "Picture changing operators in closed fermionic string field theory," Phys. Lett. B 286, 256 (1992) arXiv:hep-th/9202087.

[9] Y. Okawa and B. Zwiebach, "Heterotic string field theory," JHEP 0407, 042 (2004) arXiv:hep-th/0406212.

[10] E. Witten, "Nonabelian Bosonization In Two Dimensions," Commun. Math. Phys. 92, 455 (1984).

[11] D. J. Gross, J. A. Harvey, E. J. Martinec and R. Rohm, "Heterotic String Theory. 1. The Free Heterotic String," Nucl. Phys. B 256, 253 (1985); D. J. Gross, J. A. Harvey, E. J. Martinec and R. Rohm, "Heterotic String Theory. 2. The Interacting Heterotic String," Nucl. Phys. B 267, 75 (1986); D. J. Gross and V. Periwal, "Heterotic String Light Cone Field Theory," Nucl. Phys. B 287, 1 (1987).

[12] C. Schubert, "The finite gauge transformations in closed string field theory," Lett. Math. Phys. 26, 259 (1992).

[13] H. Ooguri and C. Vafa, "Selfduality And N=2 String Magic," Mod. Phys. Lett. A 5, 1389 (1990).

[14] N. Berkovits and C. Vafa, "N=4 topological strings," Nucl. Phys. B 433, 123 (1995) arXiv:hep-th/9407190.

[15] N. Berkovits, "A new approach to superstring field theory," Fortsch. Phys. 48, 31 (2000) arXiv:hep-th/9912121.

[16] N. Berkovits and C. Vafa, "On the Uniqueness of string theory," Mod. Phys. Lett. A 9, 653 (1994) arXiv:hep-th/9310170.

[17] H. Ooguri and C. Vafa, "N=2 heterotic strings," Nucl. Phys. B 367, 83 (1991).

[18] N. Berkovits, "The tachyon potential in open Neveu-Schwarz string field theory," JHEP 0004, 022 (2000) arXiv:hep-th/0001084; N. Berkovits, A. Sen and B. Zwiebach, "Tachyon condensation in superstring field theory," Nucl. Phys. B 587, 147 (2000) arXiv:hep-th/0002211; P.-J. De Smet and J. Raeymaekers, "Level four approximation to the tachyon potential in superstring field theory," JHEP 0005, 051 (2000) arXiv:hep-th/0003220; A. Iqbal and A. Naqvi, "Tachyon condensation on a non-BPS Dbrane," arXiv:hep-th/0004015 P.-J. De Smet, "Tachyon condensation: Calculations in string field theory," arXiv:hep-th/0109182

[19] A. Adams, J. Polchinski and E. Silverstein, "Don't panic! Closed string tachyons in ALE spacetimes," JHEP 0110, 029 (2001) arXiv:hep-th/0108075; C. Vafa, "Mirror symmetry and closed string tachyon condensation," arXiv:hep-th/0111051 J. A. Harvey, D. Kutasov, E. J. Martinec and G. Moore, "Localized tachyons and RG flows," arXiv:hep-th/0111154 A. Dabholkar, "Tachyon condensation and black hole entropy," Phys. Rev. Lett. 88, 091301 (2002) arXiv:hep-th/0111004; J. R. David, "Unstable magnetic fluxes in heterotic string theory," JHEP 0209, 006 (2002) arXiv:hep-th/0208011; Y. Okawa and B. Zwiebach, "Twisted tachyon condensation in closed string field theory," JHEP 0403, 056 (2004) arXiv:hep-th/0403051; A. Dabholkar, A. Iqubal and J. Raeymaekers, "Off-shell interactions for closed-string tachyons," JHEP 0405, 051 (2004) arXiv:hep-th/0403238; M. Headrick, S. Minwalla and T. Takayanagi, "Closed string tachyon condensation: An overview," Class. Quant. Grav. 21, S1539 (2004) arXiv:hep-th/0405064. 